\title{
BMJ Open Prompt closure versus gradual weaning of external ventricular drainage for hydrocephalus in adult patients with aneurysmal subarachnoid haemorrhage: a systematic review
}

Tenna Capion (10 , ${ }^{1}$ Alexander Lilja-Cyron, ${ }^{1}$ Marianne Juhler, ${ }^{1}$ Tiit Illimar Mathiesen, ${ }^{1}$ Jørn Wetterslev²

To cite: Capion T, Lilja-Cyron A, Juhler M, et al. Prompt closure versus gradual weaning of external ventricular drainage for hydrocephalus in adult patients with aneurysmal subarachnoid haemorrhage: a systematic review. BMJ Open 2020;10:e040722. doi:10.1136/ bmjopen-2020-040722

- Prepublication history and additional material for this paper is available online. To view these files, please visit the journal online (http://dx.doi.org/10. 1136/bmjopen-2020-040722).

Received 21 May 2020

Revised 17 October 2020

Accepted 12 November 2020

D) Check for updates

(c) Author(s) (or their employer(s)) 2020. Re-use permitted under CC BY-NC. No commercial re-use. See rights and permissions. Published by BMJ.

${ }^{1}$ Department of Neurosurgery, Copenhagen University Hospital Rigshospitalet, Copenhagen,

Denmark

${ }^{2}$ Copenhagen Trial Unit, Centre for Clinical Intervention Research, Rigshospitalet, Copenhagen, Denmark

Correspondence to

Dr Tenna Capion;

tenna.baek.capion@regionh.dk

\section{ABSTRACT}

Objectives To summarise the evidence on benefits and harms of prompt closure versus gradual weaning of external ventricular drainage (EVD) in patients with hydrocephalus following aneurysmal subarachnoid haemorrhage (aSAH) based on randomised clinical trials (RCTs) in humans.

Setting RCTs comparing prompt closure versus gradual weaning of EVD in adult patients with hydrocephalus following aSAH were included.

Participants Patients aged equal to or greater than 18 years with an EVD due to hydrocephalus following aSAH were eligible for inclusion.

Primary and secondary outcome measures Primary outcomes were all-cause mortality, any serious adverse event, rate of ventriculoperitoneal (VP) shunt placement and quality of life. Secondary outcomes were patients with shunt failure, hospital and neuro intensive care unit (NICU) length of stay (LOS) and complications related to treatment with an EVD. Data permitted report of rate of VP shunt placement, and hospital and NICU LOS.

Results Six studies were assessed in full text. One RCT with 81 patients was included. Rate of VP shunt placement was $63.4 \%$ in the rapid weaning group (ie, prompt closure of the EVD; 41 patients) and $62.5 \%$ in the gradual weaning group ( 40 patients; $p=0.932$ ). LOS in hospital and NICU was significantly shorter in the rapidly weaned group compared with the gradually weaned group (mean 19.1 vs 21.5 days in hospital $(p=0.03)$; and mean 14.1 vs 16.9 days in NICU ( $p=0.0002))$. Data were insufficient to conduct meta-analysis, trial sequential analysis or subgroup analysis of heterogeneity and sensitivity. One RCT is currently ongoing.

Conclusions We found insufficient evidence to favour any of the two strategies for EVD discontinuation in patients with hydrocephalus following aSAH.

PROSPERO registration number CRD42018108801.

\section{BACKGROUND}

Aneurysmal subarachnoid haemorrhage (aSAH) is a common and often devastating cerebrovascular disease accounting for
Strengths and limitations of this study

- Patient-centred outcomes.

- Rigorous assessment of bias and the risk of random errors.

- Grading of Recommendations Assessment, Development and Evaluation assessment of the quality of the total evidence.

- One included randomised clinical trial.

- Recommendations from systematic reviews may suffer from the quality of the included trials.

approximately $7 \%$ of all strokes. ${ }^{1}$ Acute hydrocephalus due to blockage of cerebrospinal fluid (CSF) circulation occurs as a common and severe complication, which is treated with an external ventricular drain (EVD) in the acute phase. An EVD enables removal of CSF and subsequently management of intracranial pressure (ICP). ${ }^{23} \mathrm{Up}$ to $37 \%$ of patients with an EVD develop chronic hydrocephalus during the course of the disease, requiring permanent diversion of CSF via a ventriculoperitoneal (VP) shunt. ${ }^{2}$ How to increase safety of EVD discontinuation and reduce the need for a VP shunt is debated. Two different strategies are typically being used to assess for dependence of drainage; prompt closure or gradual weaning of the EVD. The latter is performed by stepwise increase of drainage resistance to outflow over days. It is unknown whether these two strategies result in differentiated clinical outcomes, different risks for VP shunt placement or whether they lead to different complication rates of EVD and VP-shunt treatment.

\section{Description of the condition}

In adults, CSF production is constant at approximately $500 \mathrm{~mL} /$ day. Thus CSF 
circulation and absorption occur at a similar rate keeping the system in balance. Posthaemorrhagic hypersecretion of CSF or obstruction of CSF circulation and absorption result in hydrocephalus. ${ }^{4}$ The reported prevalence of hydrocephalus following aSAH ranges between $6 \%$ and $67 \%$, and three stages of hydrocephalus are generally recognised: acute (0-3 days after SAH), subacute (4-13 days after $\mathrm{SAH})$ and chronic $(\geq 14$ days after $\mathrm{SAH}){ }^{3}$

\section{Description of the intervention}

Scientific data to define timing and choice of strategy for discontinuation and removal of an EVD inserted to treat hydrocephalus following aSAH is sparse. In some patients circulation of CSF returns to normal within days or weeks, permitting the EVD to be removed with ICP within normal range and no further need for treatment. In other patients, chronic hydrocephalus evolves with the need for an implanted permanent drainage solution (a VP shunt $)^{5}$ which diverts CSF from the brain ventricles to the abdomen where it is absorbed. Prolonged duration of EVD treatment as seen in gradual weaning of the EVD is an attempt to await potential return of normal CSF circulation and thereby avoid a permanent shunt. However, the risk of serious and potentially fatal infection (ventriculitis, meningitis, cerebral abscess) increases with prolonged EVD treatment. Conversely, early discontinuation may involve risks associated with increased ICP and acute hydrocephalus and possibly increased risk for placement of a permanent shunt.

\section{How the intervention might work}

The process of identifying patients who will need a permanent VP shunt involves a trial of closure of the EVD. The main argument in favour of prompt closure of the EVD is to minimise the treatment period and thereby the risk of infection. Subsequently, patients could potentially be discharged earlier from the hospital and thus begin rehabilitation sooner. The arguments in favour of weaning by gradually increasing drainage resistance involves time for reestablishment of normal CSF circulation, and thus less drastic changes in ICP with potential protection of brain tissue.

\section{Why it is important to do this review}

A possible difference between the two treatment strategies is important to identify as difference in treatment may affect patient outcomes. Insertion of a VP shunt is best defined as a surrogate outcome measure in the present context, as the indication for the procedure seems to vary throughout and the procedure is associated with risks for the patient (ie, mechanical shunt dysfunction and shunt-related infections) and increased medical costs for society as shunt complications frequently require additional hospitalisations and surgical interventions.

Previous reviews within this field have compared the two EVD discontinuation strategies in patients with hydrocephalus following aSAH via comprehensive literature searches without pre-published protocols or predefined hypotheses or data extraction plans, and without a validated rating of the available evidence. ${ }^{67}$ A review that methodologically meets the rigorous demands for systematic reviews as defined by the Preferred Reporting Items for Systematic Reviews and Meta-Analyses (PRISMA) guidelines (and 2015 PRISMA-Protocols statement) provides the highest possible impact for researchers to use in forthcoming work and investigation of this medical issue. $^{8}$

\section{Objectives}

To summarise the evidence on benefits and harms of prompt closure vs gradual weaning of EVD in patients with hydrocephalus following aSAH based on randomised clinical trial (RCT) in humans.

\section{METHODS}

\section{Criteria for considering studies for this review}

Types of studies

This systematic review was conducted in accordance with PROSPERO registration (CRD42018108801) and a pre-published protocol. ${ }^{9}$ The recommendations from the Cochrane Collaboration, the PRISMA guidelines for systematic reviews ${ }^{8}$ and the Grading of Recommendations Assessment, Development and Evaluation (GRADE) assessment were followed. ${ }^{10}$

RCTs comparing prompt closure versus gradual weaning of EVD in patients with aSAH were included in qualitative evaluations of intervention effects in this systematic review. Additionally, observational studies were included in an appendix enumerating findings of serious adverse events (SAEs).

Studies were assessed without consideration of publication status, blinding status or language. No unpublished trials or trials using quasi-randomisation were included.

\section{Types of participants}

Patients aged equal to or greater than 18 years with an EVD due to hydrocephalus following aSAH were eligible for inclusion.

\section{Types of interventions}

Interventions studied involve prompt closure, that is, the direct closure of the EVD, versus gradual weaning, that is, a gradual increase of resistance to outflow over days, of EVD due to hydrocephalus following aSAH.

\section{Types of outcome measures}

Predefined primary outcomes include death from any cause, any SAE defined according to the International Conference of Harmonisation of Good Clinical Practice (ICH-GCP) Guidelines (E6) complications and adverse events specific for EVD and VP shunt systems (clinical and radiological signs of shunt obstruction, and clinical and microbiological signs of ventriculitis and shunt infection), rate of permanent VP shunt placement and quality of life (QoL) measured with any score. 
Predefined secondary outcomes comprise number of shunt interventions following the primary shunt insertion (surgical shunt interventions for any reason) within the longest follow-up in each trial, total hospital and neuro intensive care unit (NICU) length of stay (LOS) and EVD-related complications (ventriculitis defined as positive CSF culture, clinically relevant intracranial haemorrhage requiring surgical evacuation or additional surgical procedure secondary to EVD misplacement).

\section{Search methods for identification of studies Electronic searches}

Searches were performed without language or date restrictions. The following electronic databases were searched: The Cochrane Library's Central Register of Controlled trials (CENTRAL), MEDLINE (1946 to date) (Ovid SP), EMBASE (1974 to date) (Ovid SP), LILACS (1982 to date) (BIREME), Science Citation Index Expanded (1900 to November 2018) and Conference Proceedings Citation Index-Science (1990 to November 2018) (Web of Science). The preliminary search was performed on 28 November 2018 and repeated on 20 January 2020. The search strategies can be seen in online supplemental appendix 1.

\section{Searching other resources}

Studies included in the full-text screening were hand searched for supplemental studies not registered in the electronic searches. Main authors of studies were contacted for any missed, unreported or ongoing trials and to retrieve relevant data.

\section{Data collection and analysis}

Main authors of studies included in the trial were contacted in case their publication did not contain sufficient information for risk-of-bias (ROB) assessment and data extraction of our chosen outcomes.

\section{Selection of studies}

Two review authors (TC and AL-C) independently evaluated all relevant references and provided a detailed description of included and excluded trials.

\section{Data extraction and management}

Titles and abstracts were screened in order to identify studies that were eligible. TC and AL-C independently extracted and collected data using the Covidence software (Covidence systematic review software, Veritas Health Innovation, Melbourne, Australia). We were not blinded to the author, institution or the publication source of trials. Disagreements were resolved by JW.

Review Manager Software (Review Manager (RevMan) (Computer program), V.5.3, Copenhagen: The Nordic Cochrane Centre, The Cochrane Collaboration, 2014) was used as statistical software.

\section{Assessment of ROB in included studies}

TC and AL-C independently conducted the assessment of ROB using The Cochrane Collaboration's recommended tool for assessing ROB. Disagreements were resolved by JW.

To draw conclusions about the overall ROB for an outcome, it is necessary to evaluate the trials for major sources of bias, also defined as domains (random sequence generation, allocation concealment, blinding, incomplete outcome data, selective outcome reporting and other sources of bias). The Cochrane Collaboration's recommended tool for assessing ROB is neither a scale nor a checklist but rather a domain-based evaluation. Any assessment of the overall ROB involves consideration of the relative importance of the different domains. We will present results for all outcomes including adverse events in a 'Summary of findings' (SOF) table with a GRADE assessment of the quality of evidence for the results of each outcome. ${ }^{11}$

\section{Dealing with missing data}

Main authors of included trials were contacted in order to retrieve missing data.

For further details about the handling of missing data, and for details regarding assessment of heterogeneity, reporting bias, data synthesis, meta-analysis, trial sequential analysis and subgroup and sensitivity analysis, please see published review protocol. ${ }^{9}$

\section{Grading of Recommendations Assessment, Development and Evaluation}

The GRADE approach was used to rate and assess the quality of the evidence for each outcome. ${ }^{10}$ An SOF table was produced summarising the quality of evidence for each outcome.

\section{RESULTS}

\section{Description of studies}

Results of the search

We identified 751 references via a primary search in November 2018 and an updated search in January 2020. Fifty-six references were removed as duplicates, leaving 695 to be screened for title and abstract. Of these, six studies were assessed in full text. We found one RCT which met the inclusion criteria $^{12}$ (figure 1) and one observational study to be included in appendix ${ }^{13}$ (see online supplemental appendix 2). No studies describing prompt closure versus gradual weaning of EVD in other conditions such as spontaneous intracranial haemorrhage were found.

\section{Included studies}

The included RCT by Klopfenstein $e t a l^{2}$ randomised 81 adult patients with hydrocephalus following aSAH to either rapid or gradual weaning of the EVD. A rapid wean signified prompt closure of the EVD at time of intervention, whereas gradual weaning comprised four steps of increasing drainage resistance to outflow ending at complete closure of the EVD. Of the 81 randomised patients, 41 were in the rapidly weaned group and 40 
PRISMA Flow Diagram
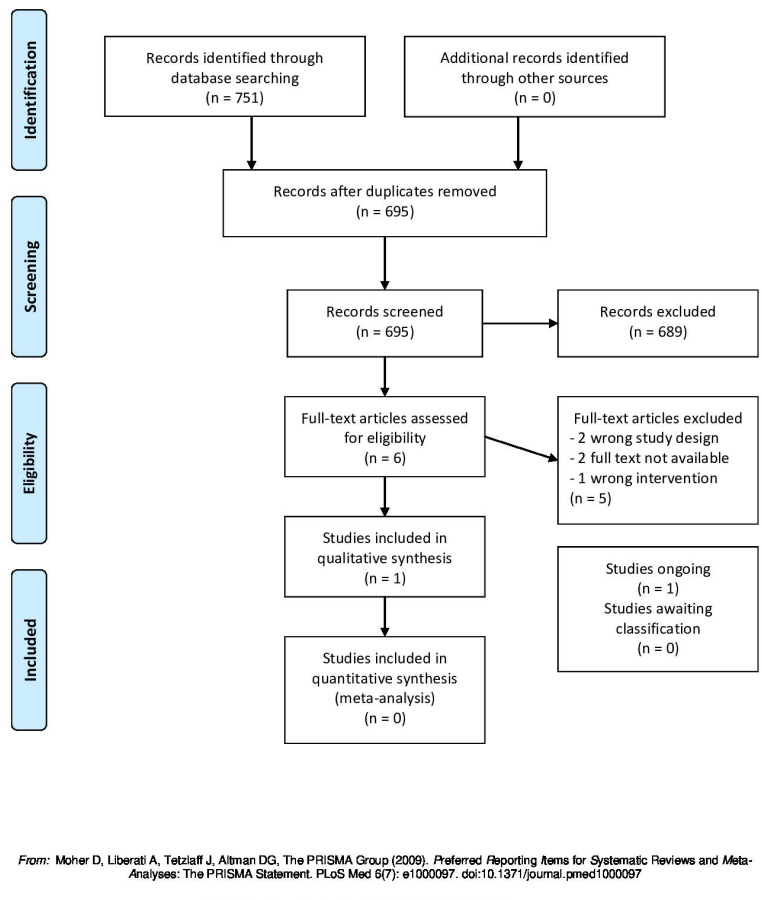

For more Information, visit www. prisma-statement.ors.

Figure 1 PRISMA flow diagram showing the results of the search. PRISMA, Preferred Reporting Items for Systematic Reviews and Meta-Analyses.

patients were in the gradually weaned group. The primary outcome of this trial was rate of VP shunt placement. Secondary outcomes were (1) number of days in which the EVD was in place; (2) number of days the patient spent in the ICU; and (3) overall duration of hospital stay.

All patients who failed either form of EVD discontinuation underwent shunt placement, resulting in equal shunt rates for the two groups. In the gradually weaned group the EVD remained in place for significantly longer time, while LOS in hospital and NICU were significantly longer for the gradually weaned group. No data were available for death by any cause, SAE or QoL at longest follow-up.

The authors concluded that gradual weaning provides no advantage over prompt closure in terms of rate of VP shunt placement, and that prompt closure should as such be pursued in the treatment of patients with aSAH due to shorter LOS in hospital and NICU and shorter time with EVD in place.

Contact by email to the corresponding and last author of this study in order to retrieve additional relevant data was attempted without result.

One observational cohort study comparing prompt closure vs gradual weaning of EVD treatment in patients with aSAH was included in an appendix enumerating adverse effects (see online supplemental appendix 2). ${ }^{13}$ The study by Jabbarli et al compared treatment effects in two individual German institutions using different discontinuation strategies for EVD in patients with aSAH. Outcomes were development and timing of shunt dependency. The authors concluded that patients treated by rapid weaning (ie, prompt closure) of the EVD had significantly higher risk of getting a VP shunt and that gradual weaning led to longer EVD treatment but not in the expense of higher risk of drain related infections.

Contact by email to the corresponding author of this study in order to retrieve additional relevant data was attempted without result.

\section{Excluded studies}

Of the six studies included in the full-text screening, five studies were excluded.

One study was excluded due to wrong study intervention. The RCT by Olson et al compared continuous versus intermittent EVD in patients with an EVD due to hydrocephalus following aSAH. The study was terminated after the inclusion of 60 patients due to a higher complication rate in the continuous drainage group. ${ }^{14}$

Two of the excluded studies were conference papers to which full texts were not available. In one of these studies, authors carried out a prospective, randomised pilot study to determine the feasibility of randomising patients with an EVD after aSAH to either aggressive or conventional CSF drainage. The authors included 20 patients of which 13 were in the aggressive arm, and concluded that randomisation is possible. The corresponding author to this study has via email informed that completion of the article was not pursued, nor was further progression with an RCT.

The second study for which full text was not available was an abstract of a retrospective assessment of 200 patients with an EVD due to non-traumatic (aneurysmal) $\mathrm{SAH}^{15}$ comparing gradual wean and early clamp trial of the EVD. The authors compared rate of VP shunt placement, NICU and hospital LOS, EVD duration and rate of EVD related infections and concluded that an early clamp trial was associated with fewer complications and shorter LOS compared with gradual weaning.

The last two references were excluded due to wrong study design; one was an observational study ${ }^{13}$ carried out in 2018 which evaluated the role of EVD weaning on rate of VP shunt placement in 965 patients with aSAH. The authors concluded that at the expense of longer treatment, gradual EVD weaning may decrease the risk of shunt dependency without an additional risk of CSF infection. The second reference omitted due to wrong study design was a comment to the study by Jabbarli $e t \mathrm{al}^{13}$, featured in the end of the article as contribution.

Details of the five excluded studies can be seen in online supplemental appendix 3 .

\section{ROB in included studies}

Using the Cochrane Collaboration's tool for assessing ROB we found that the included study had limitations in design and execution severe enough to downgrade the quality of evidence. No information regarding allocation table or concealment was provided which resulted in unclear risk of selection bias. Participants and personnel 
were not blinded to the intervention due to the nature of the intervention. Patients were randomised at time of enrolment. The timing of intervention was decided by a treating physician not involved in the trial execution but blinded to the outcome of the randomisation. No details describing how the randomisation process was performed were provided. We assessed the risk of performance bias and detection bias as high.

Outcome was reported for the 51 patients out of 81 who received a VP shunt. Follow-up status for the remaining 30 patients was not reported. Intent-to-treat analysis was described for the primary outcome but not for secondary outcomes. The numbers of eligible, included and excluded patients were provided. Reasons for patient exclusion and withdrawal were not specified neither were information about the handling of the excluded patients in terms of randomisation or intention-to-treat analysis. The risk of attrition bias was due to these limitations assessed as unclear. Further, patient-centred outcomes such as mortality, number of SAE, complications related to EVD and VP shunt treatment and QoL were not reported which made risk of reporting bias high. No study protocol was published before the study paper and no sample size calculations were provided which might have led to data driven reporting bias.

These limitations are severe in their generation of the overall ROB as they might individually and combined cause bias to the execution of the study and to the randomisation process which may cause systematic bias in the inclusion and division of patients and thus to the results of the study. Based on the assessed domains the overall ROB of the included study was assessed as high (figure 2).

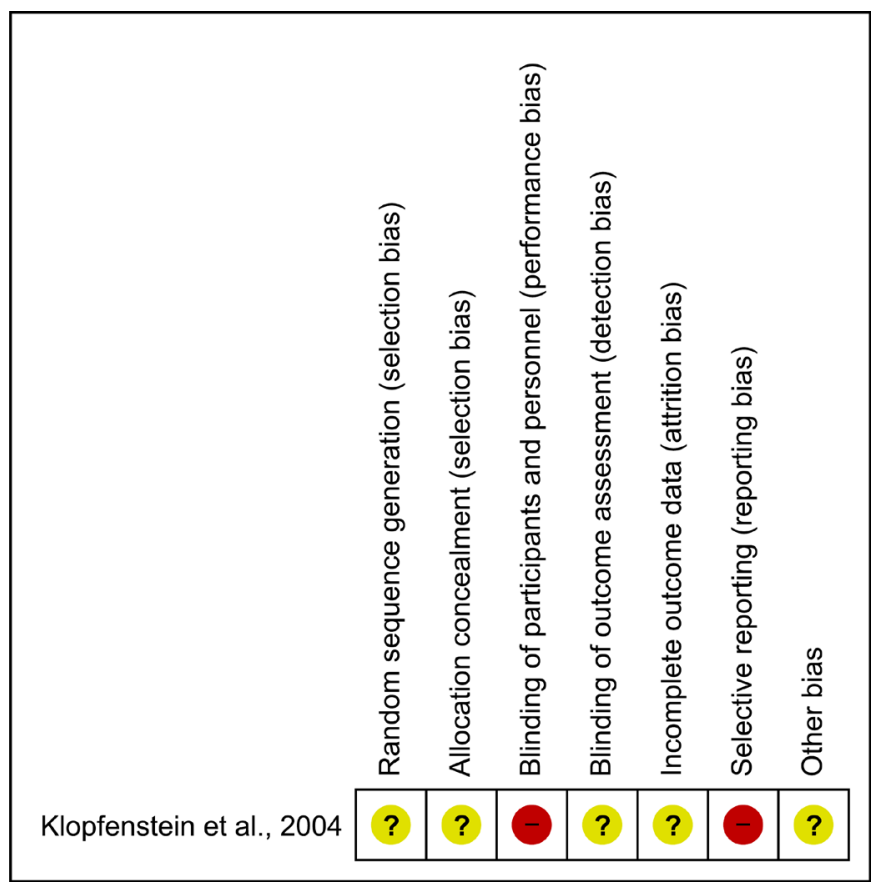

Figure 2 Risk-of-bias assessment. Red, high risk; Yellow, unclear risk.

\section{Effects of interventions}

The only primary outcome for which the included RCT provided data was rate of VP shunt placement. All patients who failed either form of EVD discontinuation underwent shunt placement, resulting in a shunt rate of $63.4 \%$ and $62.5 \%$ for rapid or gradual wean, respectively $(p=0.932)$. Certainty for this outcome assessed via the GRADE approach was considered very low primarily due to very serious ROB, very serious imprecision and serious indirectness.

Secondary outcomes for which data were available included time with EVD in place, and hospital and NICU LOS. In the rapidly weaned group the EVD remained in place for significantly shorter time compared with the gradually weaned group (mean of 12.7 vs 15.8 days, $\mathrm{p}=0.000009$ ). LOS in hospital and NICU was also shorter for the rapidly weaned group (19.1 vs 21.5 days in hospital $(\mathrm{p}=0.03) ; 14.1$ vs 16.9 days in NICU $(\mathrm{p}=0.0002))$. The certainty for these outcomes was equally considered very low based on very serious ROB, very serious imprecision and serious indirectness.

The power of the included RCT (81 patients) does not reach required information size (RIS) to conduct a reliable and conclusive meta-analysis which in size is expected to be at least that of the sample size of one wellpowered RCT for a reliable detection or rejection of an anticipated intervention effect. ${ }^{16}$ A study with few patients and few events, and thus wide CIs, raises imprecision and uncertainty about the results, as is also the result in the present included RCT.

\section{Patient and public involvement}

Patients and public were not involved in the making of this systematic review.

\section{DISCUSSION}

This systematic review aimed at assessing the evidence of benefits and harms of prompt closure versus gradual weaning of EVD in patients with hydrocephalus following aSAH. We conducted an extensive literature search which resulted in just six studies evaluated in full text. We included one RCT with 81 patients which concluded that prompt closure is safe and reduce LOS in hospital and NICU. We assessed however the RCT by Klopfenstein $e t$ $a l^{12}$ to be of overall low quality with high ROB and thus insufficient in order to provide high-quality evidence to support or refute either of the two investigated strategies for EVD discontinuation. Despite the assessed quality of the RCT, the current international guidelines covering this issue base their recommendations solely on the results from this study. ${ }^{17}$ Previous reviews differ in design and methodology, they do not assess the quality of included studies in detail, and they support the recommendations for prompt closure as discontinuation strategy despite the above mentioned shortages in evidence. ${ }^{67}$ There is currently no high-quality evidence to cover this information gap. 
The present systematic review is the first of its kind to address the question of EVD discontinuation strategy after aSAH by assessment of included studies using the Cochrane ROB tool and the GRADE approach, and it disagrees with previous review conclusions on the applicability of the results of the included RCT in international recommendations and guidelines.

\section{Summary of main results}

One RCT with 81 patients was included in this systematic review. The included trial showed very serious ROB and imprecision and an overall very low quality assessment based on the GRADE approach and the Cochrane ROB tool. RIS to provide reliable meta-analysis and TSA on our chosen outcomes was not reached and it was impossible to conduct subgroup and sensitivity analyses to investigate reasons for heterogeneity.

\section{Overall completeness and applicability of evidence}

There is insufficient evidence to favour any of the two investigated strategies for discontinuation of EVD in patients with hydrocephalus following aSAH.

\section{Quality of the evidence}

Based on GRADE the certainty of the evidence for the primary outcome and the two secondary outcomes, for which data were provided, was in all cases assessed as 'very low'. These assessments were mainly based on very serious ROB, very serious imprecision and serious indirectness (figure 3).

For the remaining three primary outcomes and two secondary outcomes which this systematic review sought to evaluate there were no available data.

\section{Potential biases in the review process}

The authors to this review has based on the preliminary literature search in November 2018 initiated and launched an RCT comparing prompt closure vs gradual weaning of external ventricular drainage in patients with hydrocephalus following aSAH which is currently ongoing. We might as such be biased in assessing methods within this field as we have previously done extensive literature search within this area of research.

\section{Agreement and disagreement with other reviews}

Chung $e$ a $\mathrm{l}^{7}$ conclude in a 2019 literature review (covering literature until 2017) that a recommendation towards an

Summary of findings:

\begin{tabular}{|c|c|c|c|c|c|c|}
\hline \multicolumn{7}{|c|}{ Prompt closure compared to gradual weaning in discontinuation of external ventricular drainage } \\
\hline \multicolumn{7}{|c|}{$\begin{array}{l}\text { Patient or population: adult patients with hydrocephalus following aneurysmal subarachnoid haemorrhage } \\
\text { Setting: discontinuation of external ventricular drainage } \\
\text { Intervention: prompt closure } \\
\text { Comparison: gradual weaning }\end{array}$} \\
\hline \multirow[b]{2}{*}{ Outcomes } & \multicolumn{2}{|c|}{ Anticipated absolute effects ${ }^{\prime}(95 \% \mathrm{Cl})$} & \multirow[b]{2}{*}{$\begin{array}{l}\text { Relative effect } \\
\quad(95 \% \mathrm{Cl})\end{array}$} & \multirow[b]{2}{*}{$\begin{array}{l}\text { № of participants } \\
\text { (studies) }\end{array}$} & \multirow{2}{*}{$\begin{array}{l}\text { Certainty of the } \\
\text { evidence } \\
\text { (GRADE) }\end{array}$} & \multirow[b]{2}{*}{ Comments } \\
\hline & $\begin{array}{l}\text { Risk with gradual } \\
\text { weaning }\end{array}$ & Risk with prompt closure & & & & \\
\hline Death - not reported & - & - & - & - & - & \\
\hline Serious Adverse Events - not reported & - & - & - & - & - & \\
\hline $\begin{array}{l}\text { Rate of permanent VP-shunt } \\
\text { implementation } \\
\text { follow up: mean } 7.5 \text { months }\end{array}$ & 63 per 100 & $\begin{array}{l}63 \text { per } 100 \\
(46 \text { to } 89)\end{array}$ & $\begin{array}{c}\text { RR } 1.01 \\
(0.73 \text { to } 1.42)\end{array}$ & $\begin{array}{c}81 \\
(1 \mathrm{RCT})\end{array}$ & $\begin{array}{l}\oplus \bigcirc \bigcirc \bigcirc \\
\text { VERY LOW } \\
a, b, c, c, d, e, f\end{array}$ & $\begin{array}{l}\text { The evidence is very uncertain about the effect of prompt } \\
\text { closure on rate of permanent VP-shunt implementation. }\end{array}$ \\
\hline Quality of life - not reported & - & - & - & - & - & \\
\hline Total hospital length of stay (LOS) & $\begin{array}{l}\text { The mean total hospital } \\
\text { length of stay was } 21.5 \\
\text { days }\end{array}$ & $\begin{array}{c}\text { mean } 2.4 \text { days lower } \\
\text { (17.1 lower to } 12.3 \text { higher) }\end{array}$ & $\cdot$ & $\begin{array}{c}81 \\
(1 \mathrm{RCT})\end{array}$ & $\begin{array}{l}\oplus \bigcirc \bigcirc \bigcirc \\
\text { VERY LOW } \\
a, b, c, c, d, e, f\end{array}$ & $\begin{array}{l}\text { Prompt closure may reduce/have little to no effect on total } \\
\text { hospital length of stay but the evidence is very uncertain. }\end{array}$ \\
\hline $\begin{array}{l}\text { Total Neuro Intensive Care Unit length of } \\
\text { stay (NICU LOS) }\end{array}$ & $\begin{array}{l}\text { The mean total Neuro } \\
\text { Intensive Care Unit length } \\
\text { of stay was } 16.9 \text { days }\end{array}$ & $\begin{array}{l}\text { mean } 2.8 \text { days lower } \\
\text { (11.4 lower to } 5.8 \text { higher) }\end{array}$ & - & $\begin{array}{c}81 \\
(1 \mathrm{RCT})\end{array}$ & $\begin{array}{l}\oplus \bigcirc \bigcirc \bigcirc \\
\text { VERY LOW } \\
\text { a,b,c,c,e,eff }\end{array}$ & $\begin{array}{l}\text { Prompt closure may reduce/have little to no effect on total } \\
\text { Neuro Intensive Care Unit length of stay but the evidence is } \\
\text { very uncertain. }\end{array}$ \\
\hline
\end{tabular}

*The risk in the intervention group (and its $95 \%$ confidence interval) is based on the assumed risk in the comparison group and the relative effect of the intervention (and its $95 \% \mathrm{Cl}$ ).

Cl: Confidence interval; RR: Risk ratio

GRADE Working Group grades of evidence

High certainty: We are very confident that the true effect lies close to that of the estimate of the effect

Moderate certainty: We are moderately confident in the effect estimate: The true effect is likely to be close to the estimate of the effect, but there is a possibility that it is substantially different

Low certainty: Our confidence in the effect estimate is limited: The true effect may be substantially different from the estimate of the effect

Very low certainty: We have very little confidence in the effect estimate: The true effect is likely to be substantially different from the estimate of effect

\section{Explanations}

a. Lack of information regarding sequence allocation and concealment of allocation table; $\mathbf{b}$. Lack of blinding; $\mathbf{c}$. Missing description of randomization; $\mathbf{d}$. Mortality and Serious Adverse Events not reported as patient important outcomes e. Very few events; f. Wide confidence intervals.

Figure 3 Summary of findings table showing the rating of the quality of the evidence for each outcome using the GRADE assessment. GRADE, grading of recommendations assessment, development and evaluation; VP, ventriculoperitoneal. 
early EVD clamp (ie, prompt closure) is possible based on the evidence of the RCT by Klopfenstein et al. ${ }^{12}$ In this literature search, the only included trial (Klopfenstein) is assessed via pragmatic evaluation and not via validated tools as the Cochrane tool for assessing ROB or the GRADE approach.

In an evidence-based consensus statement from the Neurocritical Care Society in 2015 (covering literature until 2014) the authors conclude that the RCT by Klopfenstein $e t a l^{12}$ demonstrated that rapid weaning can be accomplished safely. ${ }^{6}$ The society simultaneously underlines that the recommendation is based on one RCT with limited number of included patients. The recommendation comprises early EVD discontinuation in order to favour a decreased risk of EVD-related infections.

Our review disagrees with the conclusions of these previous reviews in the essence that we do not believe that a recommendation towards a specific weaning strategy is possible based on current available scientific data.

\section{Authors' conclusions \\ Implications of practice}

There is insufficient evidence to favour any of the two investigated strategies for EVD discontinuation in adult patients with EVD due to hydrocephalus following aSAH. Current guidelines support prompt closure of the EVD as discontinuation strategy based on the RCT described in this systematic review which has shown to be of very low quality and thus possess deficiencies severe enough to downgrade its level of evidence. Subgroup analyses were not possible to complete due to limited data and this systematic review do not allow for recommendations for clinical practice.

\section{Implications for research}

Larger, high-quality, RCTs with transparent objective criteria for randomisation, prepublished protocols to avoid data-driven reporting bias, independent sequence allocation with proper concealment and description of blinding including of outcome assessors are needed to provide reliable prospective data before conclusions regarding benefits and harms of this widely used treatment practice can be drawn safely.

Acknowledgements The group of authors would like to thank search coordinator Sarah Klingenberg from Copenhagen Trial Unit for providing the search strategy for this systematic review.

Contributors Conception or design of the work: TC, AL-C, MJ, TIM and JW. Data collection: TC, AL-C and JW. Data analysis and interpretation: TC and JW. Drafting the article: TC, AL-C and JW. Critical revision of the article: TC, AL-C, MJ, TIM and JW. Final approval of the version to be published: TC, AL-C, MJ, TIM and JW.

Funding TC has received funding from the Research Council at Copenhagen University Hospital Rigshospitalet (grant no. E-23565-03).

Declaration of interest JW has been a member of the taskforce at Copenhagen Trial Unit to develop theory, manual, and software for doing Trial Sequential Analysis presently freeware at www.ctu.dk/tsa.

Map disclaimer The funding party is not involved in the conduct of this review.

Competing interests None declared.

Patient consent for publication Not required.
Provenance and peer review Not commissioned; externally peer reviewed.

Data availability statement Data are available on reasonable request. Extracted data are available on reasonable request and only after approval from coauthors and relevant regulatory approvals.

Supplemental material This content has been supplied by the author(s). It has not been vetted by BMJ Publishing Group Limited (BMJ) and may not have been peer-reviewed. Any opinions or recommendations discussed are solely those of the author(s) and are not endorsed by BMJ. BMJ disclaims all liability and responsibility arising from any reliance placed on the content. Where the content includes any translated material, BMJ does not warrant the accuracy and reliability of the translations (including but not limited to local regulations, clinical guidelines, terminology, drug names and drug dosages), and is not responsible for any error and/or omissions arising from translation and adaptation or otherwise.

Open access This is an open access article distributed in accordance with the Creative Commons Attribution Non Commercial (CC BY-NC 4.0) license, which permits others to distribute, remix, adapt, build upon this work non-commercially, and license their derivative works on different terms, provided the original work is properly cited, appropriate credit is given, any changes made indicated, and the use is non-commercial. See: http://creativecommons.org/licenses/by-nc/4.0/.

ORCID iD

Tenna Capion http://orcid.org/0000-0002-6968-1938

\section{REFERENCES}

1 Qian C, Yu X, Chen J, et al. Effect of the drainage of cerebrospinal fluid in patients with aneurismal subarachnoid hemorrhage. Medicine 2016;95:e5140.

2 Xie Z, Hu X, Zan X, et al. Predictors of Shunt-dependent hydrocephalus after aneurysmal subarachnoid hemorrhage? A systematic review and meta-analysis. World Neurosurg 2017;106:844-60.

3 Dorai Z, Hynan LS, Kopitnik TA, a KT, et al. Factors related to hydrocephalus after aneurysmal subarachnoid hemorrhage. Neurosurgery 2003;52:763-71.

4 van Gijn J, Kerr RS, Rinkel GJE. Subarachnoid haemorrhage. The Lancet 2007;369:306-18.

5 Woernle CM, Winkler KML, Burkhardt J-K, et al. Hydrocephalus in 389 patients with aneurysm-associated subarachnoid hemorrhage. $J$ Clin Neurosci 2013;20:824-6.

6 Fried HI, Nathan BR, Rowe AS, et al. The insertion and management of external ventricular drains: an evidence-based consensus statement. Neurocrit Care 2016;24:61-81.

7 Chung DY, Mayer SA, Rordorf GA. External ventricular drains after subarachnoid hemorrhage: is less more? Neurocrit Care 2018;28:157-61.

8 Moher D, Liberati A, Tetzlaff J, et al. Preferred reporting items for systematic reviews and meta-analyses: the PRISMA statement. $J$ Clin Epidemiol 2009;62:1006-12.

9 Capion T, Lilja-Cyron A, Juhler M, et al. Prompt closure versus gradual weaning of extraventricular drainage for hydrocephalus in adult patients with aneurysmal subarachnoid haemorrhage: a systematic review protocol with meta-analysis and trial sequential analysis. BMJ Open 2019;9:e029719.

10 Guyatt GH, Oxman AD, Kunz R, et al. Grade guidelines 6. rating the quality of evidence-imprecision. J Clin Epidemiol 2011;64:1283-93.

11 Higgins J, Green S. Cochrane handbook for systematic reviews of interventions version 5.1.0, 2011http://handbook.cochrane.org

12 Klopfenstein JD, Kim LJ, Feiz-Erfan I, et al. Comparison of rapid and gradual weaning from external ventricular drainage in patients with aneurysmal subarachnoid hemorrhage: a prospective randomized trial. J Neurosurg 2004;100:225-9.

13 Jabbarli R, Pierscianek D, RÖlz R, et al. Gradual external ventricular drainage weaning reduces the risk of shunt dependency after aneurysmal subarachnoid hemorrhage: a pooled analysis. Oper Neurosurg 2018;15:498-504.

14 Olson DM, Zomorodi M, Britz GW, et al. Continuous cerebral spinal fluid drainage associated with complications in patients admitted with subarachnoid hemorrhage. J Neurosurg 2013;119:974-80.

15 Rao SS, Rordorf GA. Abstracts presented at the neurocritical care Society (NCS) 15th annual meeting. Neurocrit Care;27:1-491.

16 Wetterslev J, Thorlund K, Brok J, et al. Estimating required information size by quantifying diversity in random-effects model meta-analyses. BMC Med Res Methodol 2009;9:1-12.

17 Connolly ES, a RA, Carhuapoma JR, et al. Guidelines for the management of aneurysmal subarachnoid hemorrhage: a guideline 
